\title{
Volume flexible multi items inventory system with imprecise environment
}

\author{
Surbhi Singhal* and S.R. Singh
}

Department Of Mathematics, D.N. College, Meerut, India

\begin{tabular}{l}
\hline C H R O N I C L E \\
\hline Article history: \\
Received June 12013 \\
Received in revised format \\
July $15 \quad 2013$ \\
Accepted July 152013 \\
Available online \\
July 152013 \\
\hline Keywords: \\
Volume flexible \\
Imprecise method \\
Multi items \\
Machine breakdown \\
Variable holding cost \\
\hline
\end{tabular}

\section{A B S T R A C T}

\begin{abstract}
This paper addresses a multi items volume flexible system for time dependent decaying items with the concept of machine breakdown and imprecise environment. In this study, partially backlogged shortages have been discussed. All the costs are fuzzified with signed distance method. Numerical examples are given to illustrate the theoretical results and sensitivity analysis is given to validate the results for various parameters.
\end{abstract}

\section{Introduction}

Most of the studies have taken into consideration only the crisp aspect of inventory models. In crisp studies, all the parameters of the model have definite values and there is no uncertainty associated with them. However, in reality, there are some uncertainties also about the values of different cost parameters involved in the system. For any particular problem in the crisp scenario, the aim is to maximize or minimize the objective function under the given constraints. However, in many practical situations, the decision maker may not be in a position to specify the objective or the constraints, precisely, but rather may specify them in the "fuzzy sense". In such a case, the decision maker has to depend on the fuzzy methods of solving the problem. Fuzzification grants authenticity to the model in the sense that it allows vagueness in the whole setup which brings it closer to reality.

In a perfect competitive situation, the holding cost, shortages cost, lost sale cost and the set-up cost may fluctuate a little from its actual value in the different replenishment cycles. These are some 
uncertainties in inventory optimization tasks that cannot be treated appropriately by usual probabilistic models. Therefore, it is realistic to consider the idea of fuzzy.

In 1965, the first publication in fuzzy set theory by Zadeh (1965) showed the intention to accommodate uncertainty in the non-stochastic sense. Zimmermann (1985) developed a tolerance approach to transform a fuzzy decision making problem to a regular crisp optimization problem and showed that it could be solved to obtain a unique exact optimal solution with highest membership degree using classical optimization algorithms. Padmanabhan and Vrat (1990) formulated an inventory problem of deteriorating items with two objectives of minimization of total average cost and wastage cost in crisp environment. Goyal (1991) discussed a procedure for determining the economic manufacturing policy for a product and the economic ordering policy for the raw materials required by the product. The demand for the product was constant over time and shortages were not permitted. Tse and Makis (1994) considered preventive replacement with two types of machine failure - major and minor. When a major failure occurs, the failed unit is replaced by a new one and the interrupted lot was aborted. They have used the concept of crisp. Srinivasan and Lee (1996) developed a manufacturing model when the demand rate was taken as variable, processing time follows an arbitrary probability distribution. Roy and Maiti (1998) formulated an inventory problem of deteriorating items with two objectives, namely, maximizing total average profit and minimizing total cost in fuzzy model. Roy and Maiti (2000), Mahapatra and Maiti (2005) have solved the classical order level inventory models in fuzzy environment. Yao and $\mathrm{Wu}(1999)$ and Dey et al. (2005) have considered the production model with fuzzy environment. Goyal and Giri (2003) developed the production-inventory problem in which the demand, production and deterioration rates of a product were assumed to vary with time. Giri and Dohi (2005) presented an exact formulation of EMQ model under a general framework in which the time to machine failure, corrective and preventive repair times all are assumed to follow arbitrary probability distributions. Wee and Chung (2005) considered an optimal production model for deteriorating items with constant demand. The objective was to determine the inventory models to maximize the net benefit of the production system. In their study, deterioration rate was constant and shortages were not allowed. Singh et al. (2008) developed an inventory model for decaying items with variable demand and variable holding cost in crisp environment. Maity and Maiti (2008) developed the optimal production policy for an inventory control system of deteriorating items in fuzzy environment. Here, the deterioration of the items and depreciation of sales were at a constant rate. Singh and Singh (2008) considered the fuzzy inventory model for finite rate of replenishment using signed distance method. Chakraborty et al. (2008) presented a generalized economic manufacturing quantity model for an unreliable production system in which the production facility may shift from an 'in-control' state to an 'out-of-control' state at any random time and may ultimately break down afterwards. Chakraborty et al. (2009) developed a production inventory system for deteriorating items in which the production facility may not only shift from an 'in-control' state to an 'out-of-control' state. Xu and Zhai (2010) discussed the coordination problem for single-period supply chain problems with fuzzy demand. Mahata and Mahata (2011) considered the EOQ model for a retailer to reflect the inventory system situation in the fuzzy sense. Wang et al. (2012) developed continuous review inventory models in which a fraction of demand is backordered and the remaining fraction is lost during the stock out period under fuzzy demands. Singh et al. (2013) discussed a centralized reverse channel structure with flexible manufacturing under the stock out situation. Shukla et al. (2013) considered EOQ model for deteriorating items with exponential demand rate and shortages. Hsu (2012) discussed an EOQ for items with imperfect quality and inspection errors.

This paper investigated an Economic Manufacturing Quantity model for time dependent decaying items and selling price demand with volume flexible and fuzzy environment. We assumed that different machines ' $A_{i}$ ' $(1,2 \ldots . n)$ are dedicated to the production of different items ' $i$ ' with different production rates ' $\mathrm{P}_{\mathrm{i}}$ '. The management of production in machine $\mathrm{A}_{\mathrm{i}}$ is vested with the management unit ' $\mathrm{B}_{\mathrm{i}}$ '. It is assumed that a machine may become out of order during its working time. As a result, there is a mean time for every machine between its failure/breakdowns. During a breakdown of a machine, there is 
demand although there is no production. In such a situation, the demand is met until the inventory level falls below the quantity demanded. When inventory level becomes less than the demand, the concerned management unit $B_{i}$ is rendered fully idle. This situation occurs when the customer is a wholesaler having the demand of a big lot size and the concerned management unit can't meet this demand because the stock size is less than the quantity demanded. Therefore, we considered the idle time of each management unit; this idle time leads to an additional cost for the last man hours. We have considered the capital available for manufacturing the items is limited. Shortages are allowed with partial backlogging. After that a numerical example is provided and sensitivity analysis is carried out to demonstrate the significance of considering profit maximization technique.

\section{Assumptions and Notations}

The mathematical models in this study are developed on the basis of the following assumptions and notations:

\section{Assumptions}

The following assumptions for the proposed model, which are as follows:

1. The model is developed for multiple items.

2. The demand for the item is a downward slopping function of the price.

3. Deterioration rate is time dependent.

4. Production rate per unit time is considered as a decision variable.

5. Invested capital for production is limited.

6. Machine breakdown is considered during the production period.

7. Idle time to the management unit is considered.

8. Unit production cost for the $i^{t h}$ item $(i=1,2,3 \ldots, n)$ is a function of the production rate.

9. Holding cost is a linear increasing function of time.

10. Shortages are allowed with partial backlogging during the idle time.

11. Backlogging rate is taken as constant.

12. Time horizon is infinite.

\section{Notations}

The following notations are assumed in our study:

$\mathrm{Q}_{\mathrm{i}}(\mathrm{t}) \quad$ The on-hand inventory of the $\mathrm{i}^{\text {th }}$ item at time $t$

$\mathrm{P}_{\mathrm{i}} \quad$ The production rate per unit time for the $\mathrm{i}^{\text {th }}$ item

$\theta_{i} t \quad$ Time dependent deterioration rate $0<\theta_{i}<1$

$\mu_{i} \quad$ The mean time between successive breakdowns of the machines $\left(A_{i}, i=1,2 \ldots n\right)$

$\psi_{i}\left(t_{i}\right)$ Probability density function of $t_{i}$

C Unit purchasing cost with $C<S_{p}{ }^{i}$

$m_{i} \quad$ The mean time of repair of $i^{\text {th }}$ machine

$\tau_{i} \quad$ The mean duration of a breakdown of machines $\left(A_{i}, i=1,2 \ldots, n\right)$

$\phi_{i}\left(\tau_{i}\right) \quad$ Probability density function of $\tau_{i}$

$C_{h}{ }^{i}+\gamma t \quad$ The cost of carrying one unit of $i^{t h}$ item in inventory per unit time

$C_{S}{ }^{i} \quad$ The cost of shortages per unit time of $i^{\text {th }}$ item

$C_{L S}{ }^{i} \quad$ The cost of lost sale per unit time of $i^{\text {th }}$ item 
$\eta_{i}\left(P_{i}\right) \quad$ The cost for production of a unit time of the $i^{\text {th }}$ item $(i=1,2 \ldots, n)$ and $\eta_{i}\left(P_{i}\right)=\left(r_{i}+\frac{g_{i}}{P_{i}}+\alpha_{i} P_{i}\right)$, where $\mathrm{r}_{\mathrm{i}}$ is material cost, $\alpha_{i}$ is tool or die cost and $\mathrm{g}_{\mathrm{i}}$

is energy and labor cost

$S_{p}{ }^{i} \quad$ The selling price per unit of $\mathrm{i}^{\text {th }}$ item

$W_{i} \quad$ The cost per unit of idle time of the management unit $\mathrm{B}_{\mathrm{i}}$

CAP The total capital available for production of all the items

$\mathrm{D}_{\mathrm{i}} \quad$ The annual demand as a decreasing function of price; $D_{i}\left(S_{p}{ }^{i}\right)=\alpha_{i}\left(S_{p}{ }^{i}\right)^{-\beta_{i}}$,

Where $\alpha_{i}>0$ and $\beta_{i}>1$

\section{Formulation of the Model}

In this model a multi-items inventory system with machine breakdown and volume flexibility has been considered. The production cycle begins with zero stock. Production starts at time $t=0$ and the stock reaches a level $Q_{i}\left(t_{i}\right)\{i=1,2 \ldots \ldots n\}$ at times $t=t_{i}$ after adjusting demand rates $D_{i}$. At times $t=t_{i}$ machines $\mathrm{A}_{\mathrm{i}}$, becomes out of order. Then repairing of machines $\mathrm{A}_{\mathrm{i}}$ starts and takes times $\tau_{i}$ to comeback into working state. Here, $t_{i}$ and $\tau_{i}$ are random variables which follow probability distribution functions $\psi_{i}$ and $\Phi_{i}$, respectively. The governing equations for the inventory system are as follows:

$$
\begin{array}{lrl}
Q_{i}^{\prime}(t)+\theta_{i} t Q_{i}(t) & =P_{i}-\alpha_{i}\left(S_{p}{ }^{i}\right)^{-\beta_{i}} & 0 \leq t \leq t_{i} \\
Q_{i}^{\prime}(t)+\theta_{i} t Q_{i}(t) & =-\alpha_{i}\left(S_{p}{ }^{i}\right)^{-\beta_{i}} & t_{i} \leq t \leq x
\end{array}
$$

With the boundary condition $Q_{i}(0)=0$ and $Q_{i}(x)=0$, where $\mathrm{i}=1,2 \ldots \ldots$, solution of the Eq. (1) and

Eq. (2) are:

$$
\begin{array}{ll}
Q_{i}(t)=\left(P_{i}-\alpha_{i}\left(S_{p}{ }^{i}\right)^{-\beta_{i}}\right)\left(t+\frac{\theta_{i} t^{3}}{6}\right) e^{-\frac{\theta_{i} t^{2}}{2}} & 0 \leq t \leq t_{i} \\
Q_{i}(t)=\alpha_{i}\left(S_{p}{ }^{i}\right)^{-\beta_{i}}\left[(x-t)+\frac{\theta_{i}}{6}\left(x^{3}-t^{3}\right)\right] e^{-\frac{\theta_{t} t^{2}}{2}} & t_{i} \leq t \leq x
\end{array}
$$

The idle times of the management units $\left\{B_{i}, i=1,2 \ldots \ldots n\right\}$ due to a breakdown of the machines $\left\{A_{i}\right.$, $i=1,2 \ldots \ldots n\}$ are

$$
\left\{\begin{array}{l}
\text { if } \frac{Q_{i}\left(t_{i}\right)}{\alpha_{i}\left(S_{p}\right)^{-\beta_{i}}} \geq \tau_{i} \quad u_{i}=0 \\
\text { if } \frac{Q_{i}\left(t_{i}\right)}{\alpha_{i}\left(S_{p}\right)^{-\beta_{i}}}<\tau_{i} \quad u_{i}=\tau_{i}-\frac{Q_{i}\left(t_{i}\right)}{\alpha_{i}\left(S_{p}{ }^{i}\right)^{-\beta_{i}}}
\end{array}\right.
$$

The expected cost per breakdown of the machine $\left\{A_{i}, i=1,2 \ldots \ldots n\right\}$ during the idle time is given by:

$$
\begin{aligned}
& E_{i c}{ }^{i}=W_{i} \int_{0}^{\infty}\left[\int_{\frac{Q_{i}\left(t_{i}\right)}{\alpha_{i}\left(S_{p}^{i}\right)^{-\beta_{i}}}}^{\infty}\left(\tau_{i}-\frac{Q_{i}\left(t_{i}\right)}{\alpha_{i}\left(S_{p}{ }^{i}\right)^{-\beta_{i}}}\right) \phi_{i}\left(\tau_{i}\right) d \tau_{i}\right] \psi_{i}\left(t_{i}\right) d t_{i} \\
& =\frac{W_{i}}{\mu_{i}}\left(\frac{P_{i}}{\alpha_{i}\left(S_{p}{ }^{i}\right)^{-\beta_{i}}}-1\right)\left[\mu_{i}{ }^{2}-30 \theta_{i} \mu_{i}{ }^{3}-\left(P_{i}-\alpha_{i}\left(S_{p}{ }^{i}\right)^{-\beta_{i}}\right)\left(\frac{2 \mu_{i}^{3}}{m_{i} \alpha_{i}\left(S_{p}{ }^{i}\right)^{-\beta_{i}}}-\frac{4 \mu_{i}^{3}}{m_{i} \alpha_{i}\left(S_{p}{ }^{i}\right)^{-\beta_{i}}}\right)\right]
\end{aligned}
$$

The expected shortage cost for $i^{\text {th }}$ item during the idle time is

$$
E_{S c}{ }^{i}=C_{S}{ }^{i} \alpha_{i}\left(S_{p}{ }^{i}\right)^{-\beta_{i}} \int_{0}^{\infty}\left[\int_{\frac{Q_{i}\left(t_{i}\right)}{\alpha_{i}\left(S_{p}{ }^{i}\right)^{-\beta_{i}}}}^{\infty}\left(\tau_{i}-\frac{Q_{i}\left(t_{i}\right)}{\alpha_{i}\left(S_{p}{ }^{i}\right)^{-\beta_{i}}}\right) \phi_{i}\left(\tau_{i}\right) d \tau_{i}\right] \psi_{i}\left(t_{i}\right) d t_{i}
$$




$$
=C_{S}^{i}\left[\alpha_{i}\left(S_{p}^{i}\right)^{-\beta_{i}} m_{i}-\left(P_{i}-\alpha_{i}\left(S_{p}^{i}\right)^{-\beta_{i}}\right) \mu_{i}+2\left(P_{i}-\alpha_{i}\left(S_{p}{ }^{i}\right)^{-\beta_{i}}\right) \theta_{i} \mu_{i}{ }^{3}\right]
$$

The expected lost sale cost for $i^{\text {th }}$ item during the idle time is given by:

$$
\begin{aligned}
E_{S c}{ }^{i} & =C_{L S}{ }^{i} \int_{0}^{\infty}\left[\int_{\frac{Q_{i}\left(t_{i}\right)}{\alpha_{i}\left(S_{p}\right)^{-\beta_{i}}}}^{\infty}(1-\delta) \alpha_{i}\left(S_{p}{ }^{i}\right)^{-\beta_{i}} \phi_{i}\left(\tau_{i}\right) d \tau_{i}\right] \psi_{i}\left(t_{i}\right) d t_{i} \\
& =\frac{C_{L S}{ }^{i}(1-\delta) \alpha_{i}\left(S_{p}{ }^{i}\right)^{-\beta_{i}}}{\mu_{i}}\left[\mu_{i}-\frac{\left(P_{i}-\alpha_{i}\left(S_{p}{ }^{i}\right)^{-\beta_{i}}\right)}{m_{i} \alpha_{i}\left(S_{p}{ }^{i}\right)^{-\beta_{i}}}\left(\mu_{i}{ }^{2}+\frac{\theta_{i} \mu_{i}^{3}}{3}\right)\right]
\end{aligned}
$$

Now, the total inventory of $i^{\text {th }}$ item is as follows:

$\operatorname{In} v_{i}\left(t_{i}\right)=$ Inventory during $\left[0, t_{i}\right]+$ Inventory during $\left[t_{i}, x\right]$

$$
\begin{aligned}
\operatorname{Inv} v_{i}\left(t_{i}\right)= & {\left[\int_{0}^{t_{i}}\left(C_{h}{ }^{i}+\gamma t\right) Q_{i}\left(t_{i}\right) d t_{i}+\int_{t_{i}}^{x}\left(C_{h}{ }^{i}+\gamma t\right) Q_{i}\left(t_{i}\right) d t_{i}\right] } \\
= & \left(P_{i}-\alpha_{i}\left(S_{p}{ }^{i}\right)^{-\beta_{i}}\right)\left[C_{h}{ }^{i}\left(\frac{t_{i}{ }^{2}}{2}-\frac{\theta_{i} t_{i}{ }^{4}}{12}\right)+\gamma\left(\frac{t_{i}{ }^{3}}{3}-\frac{\theta_{i} t_{i}{ }^{5}}{15}\right)\right]+\alpha_{i}\left(S_{p}{ }^{i}\right)^{-\beta_{i}}\left[C _ { h } { } ^ { i } \left(\frac{\left(P_{i}-\alpha_{i}\left(S_{p}{ }^{i}\right)^{-\beta_{i}}\right)^{2} t_{i}{ }^{2}}{2 \alpha_{i}\left(S_{p}{ }^{i}\right)^{-2 \beta_{i}}}\right.\right. \\
& -\frac{\left(P_{i}-\alpha_{i}\left(S_{p}{ }^{i}\right)^{-\beta_{i}}\right) t_{i}{ }^{2}}{\alpha_{i}\left(S_{p}{ }^{i}\right)^{-\beta_{i}}}+\frac{t_{i}{ }^{2}}{2}-\frac{\left(P_{i}-\alpha_{i}\left(S_{p}{ }^{i}\right)^{-\beta_{i}}\right)^{3} \theta_{i} t_{i}{ }^{4}}{6 \alpha_{i}\left(S_{p}{ }^{i}\right)^{-3 \beta_{i}}}-\frac{\left(P_{i}-\alpha_{i}\left(S_{p}{ }^{i}\right)^{-\beta_{i}}\right)^{4} \theta_{i} t_{i}{ }^{4}}{12 \alpha_{i}\left(S_{p}{ }^{i}\right)^{-4 \beta_{i}}}+\frac{\theta_{i} t_{i}{ }^{4}}{12} \\
& \left.+\frac{\left(P_{i}-\alpha_{i}\left(S_{p}{ }^{i}\right)^{-\beta_{i}}\right) \theta_{i} t_{i}{ }^{4}}{6 \alpha_{i}\left(S_{p}{ }^{i}\right)^{-\beta_{i}}}\right)+\gamma\left(\frac{\left(P_{i}-\alpha_{i}\left(S_{p}{ }^{i}\right)^{-\beta_{i}}\right)^{3} t_{i}{ }^{3}}{3 \alpha_{i}\left(S_{p}{ }^{i}\right)^{-3 \beta_{i}}}+\frac{t_{i}^{3}}{6}-\frac{\left(P_{i}-\alpha_{i}\left(S_{p}{ }^{i}\right)^{-\beta_{i}}\right) t_{i}{ }^{4}}{2 \alpha_{i}\left(S_{p}{ }^{i}\right)^{-\beta_{i}}}+\frac{\theta_{i} t_{i}{ }^{5}}{15}\right. \\
& \left.\left.+\frac{\left(P_{i}-\alpha_{i}\left(S_{p}{ }^{i}\right)^{-\beta_{i}}\right)^{5} \theta_{i} t_{i}{ }^{5}}{40 \alpha_{i}\left(S_{p}{ }^{i}\right)^{-5 \beta_{i}}}-\frac{\left(P_{i}-\alpha_{i}\left(S_{p}{ }^{i}\right)^{-\beta_{i}}\right)^{3} \theta_{i} t_{i}{ }^{5}}{6 \alpha_{i}\left(S_{p}{ }^{i}\right)^{-3 \beta_{i}}}+\frac{\left(P_{i}-\alpha_{i}\left(S_{p}{ }^{i}\right)^{-\beta_{i}}\right) \theta_{i} t_{i}{ }^{5}}{8 \alpha_{i}\left(S_{p}{ }^{i}\right)^{-\beta_{i}}}\right)\right]
\end{aligned}
$$

As a result, the expected inventory cost for $i^{\text {th }}$ item is as follows:

$$
\begin{aligned}
E_{\text {inc }}{ }^{i}= & \int_{0}^{\infty} \operatorname{Inv}_{i}\left(t_{i}\right) \psi_{i}\left(t_{i}\right) d t_{i} \\
= & \frac{\left(P_{i}-\alpha_{i}\left(S_{p}{ }^{i}\right)^{-\beta_{i}}\right)}{\mu_{i}}\left[C_{h}{ }^{i}\left(\mu_{i}{ }^{3}-2 \theta_{i} \mu_{i}{ }^{4}\right)+\gamma\left(2 \mu_{i}{ }^{3}-8 \mu_{i}{ }^{5}\right)\right]+\alpha_{i}\left(S_{p}{ }^{i}\right)^{-\beta_{i}}\left[C _ { h } { } ^ { i } \left(\frac{\left(P_{i}-\alpha_{i}\left(S_{p}{ }^{i}\right)^{-\beta_{i}}\right)^{2} \mu_{i}{ }^{3}}{\alpha_{i}\left(S_{p}{ }^{i}\right)^{-2 \beta_{i}}}\right.\right. \\
& -\frac{2\left(P_{i}-\alpha_{i}\left(S_{p}{ }^{i}\right)^{-\beta_{i}}\right) \mu_{i}{ }^{3}}{\alpha_{i}\left(S_{p}{ }^{i}\right)^{-\beta_{i}}}+\mu_{i}{ }^{3}-\frac{4\left(P_{i}-\alpha_{i}\left(S_{p}{ }^{i}\right)^{-\beta_{i}}\right)^{3} \theta_{i} \mu_{i}{ }^{4}}{\alpha_{i}\left(S_{p}{ }^{i}\right)^{-\beta_{i}}}-\frac{2\left(P_{i}-\alpha_{i}\left(S_{p}{ }^{i}\right)^{-\beta_{i}}\right)^{4} \theta_{i} \mu_{i}{ }^{4}}{\alpha_{i}\left(S_{p}{ }^{i}\right)^{-4 \beta_{i}}}+2 \theta_{i} \mu_{i}{ }^{4} \\
& \left.-\frac{4\left(P_{i}-\alpha_{i}\left(S_{p}{ }^{i}\right)^{-\beta_{i}}\right) \theta_{i} \mu_{i}{ }^{4}}{\alpha_{i}\left(S_{p}{ }^{i}\right)^{-\beta_{i}}}\right)+\gamma\left(\frac{2\left(P_{i}-\alpha_{i}\left(S_{p}{ }^{i}\right)^{-\beta_{i}}\right)^{3} \mu_{i}{ }^{4}}{\alpha_{i}\left(S_{p}{ }^{i}\right)^{-3 \beta_{i}}}+\mu_{i}{ }^{4}-\frac{3\left(P_{i}-\alpha_{i}\left(S_{p}{ }^{i}\right)^{-\beta_{i}}\right) \mu_{i}{ }^{4}}{\alpha_{i}\left(S_{p}{ }^{i}\right)^{-\beta_{i}}}+\frac{3\left(P_{i}-\alpha_{i}\left(S_{p}{ }^{i}\right)^{-\beta_{i}}\right)^{5} \theta_{i} \mu_{i}{ }^{5}}{\alpha_{i}\left(S_{p}{ }^{i}\right)^{-5 \beta_{i}}}\right. \\
& \left.\left.+\frac{\theta_{i} \mu_{i}{ }^{5}}{15}-\frac{20\left(P_{i}-\alpha_{i}\left(S_{p}{ }^{i}\right)^{-\beta_{i}}\right)^{3} \theta_{i} \mu_{i}{ }^{5}}{\alpha_{i}\left(S_{p}{ }^{i}\right)^{-3 \beta_{i}}}+15\left(P_{i}-\alpha_{i}\left(S_{p}{ }^{i}\right)^{-\beta_{i}}\right) \theta_{i} \mu_{i}{ }^{5}\right)\right]
\end{aligned}
$$

The, production cost per unit of $i^{\text {th }}$ item is given by:

$\eta_{i}\left(P_{i}\right)=r_{i}+\frac{g_{i}}{P_{i}}+\alpha_{i} P_{i}$

For that reason, the expected production cost for $i^{\text {th }}$ item is given by:

$$
E_{p r c}{ }^{i}=\sum_{i=1}^{n} \int_{0}^{\infty} \eta_{i}\left(P_{i}\right) P_{i} t_{i} \psi_{i}\left(t_{i}\right) d t_{i}=\sum_{i=1}^{n}\left(r_{i}+\frac{g_{i}}{P_{i}}+\alpha_{i} P_{i}\right) P_{i} \mu_{i}
$$

Then the expected set up cost for $i^{\text {th }}$ item, which is as follows:

$$
S E_{U P}^{i}=\sum_{i=1}^{n} \int_{0}^{\infty} S_{U P}^{i} t_{i} \psi_{i}\left(t_{i}\right) d t_{i}=\sum_{i=1}^{n} S_{U P}{ }^{i}
$$


The reliability of spare parts of a machine follows exponential probability distribution function. The probability density functions of exponential distribution is as follows

$\psi_{i}\left(t_{i}\right)=\frac{1}{\mu_{i}} e^{-t_{i} / \mu_{i}}$

$\phi_{i}\left(\tau_{i}\right)=\frac{1}{m_{i}} e^{-\tau_{i} / m_{i}}$

The expected total profit per breakdown is given by:

$\operatorname{ETP}\left(P_{1}, P_{2} \ldots . . P_{n}\right)=$ Expected revenue from selling items- Expected production cost - Expected holding cost-Expected cost for idle time-Expected shortages cost-Expected lost sale cost - Expected set up cost

$$
\begin{aligned}
& =\sum_{i=1}^{n}\left[\left(S_{p}^{i}-\left(r_{i}+\frac{g_{i}}{P_{i}}+H_{i} P_{i}\right)\right) P_{i} \mu_{i}-\frac{\left(P_{i}-\alpha_{i}\left(S_{p}^{i}\right)^{-\beta_{i}}\right)}{\mu_{i}}\left\{C_{h}^{i}\left(\mu_{i}^{3}-2 \theta_{i} \mu_{i}^{4}\right)+\gamma\left(2 \mu_{i}^{3}-8 \mu_{i}^{5}\right)\right\}\right. \\
& -\alpha_{i}\left(S_{p}{ }^{i}\right)^{-\beta_{i}}\left\{C _ { h } { } ^ { i } \left(\frac{\left(P_{i}-\alpha_{i}\left(S_{p}{ }^{i}\right)^{-\beta_{i}}\right)^{2} \mu_{i}^{3}}{\alpha_{i}\left(S_{p}{ }^{i}\right)^{-2 \beta_{i}}}-\frac{2\left(P_{i}-\alpha_{i}\left(S_{p}{ }^{i}\right)^{-\beta_{i}}\right) \mu_{i}^{3}}{\alpha_{i}\left(S_{p}{ }^{i}\right)^{-\beta_{i}}}+\mu_{i}{ }^{3}-\frac{4\left(P_{i}-\alpha_{i}\left(S_{p}{ }^{i}\right)^{-\beta_{i}}\right)^{3} \theta_{i} \mu_{i}{ }^{4}}{\alpha_{i}\left(S_{p}{ }^{i}\right)^{-3 \beta_{i}}}\right.\right. \\
& \left.-\frac{2\left(P_{i}-\alpha_{i}\left(S_{p}{ }^{i}\right)^{-\beta_{i}}\right)^{4} \theta_{i} \mu_{i}{ }^{4}}{\alpha_{i}\left(S_{p}{ }^{i}\right)^{-4 \beta_{i}}}+2 \theta_{i} \mu_{i}{ }^{4}-\frac{4\left(P_{i}-\alpha_{i}\left(S_{p}{ }^{i}\right)^{-\beta_{i}}\right) \theta_{i} \mu_{i}{ }^{4}}{\alpha_{i}\left(S_{p}{ }^{i}\right)^{-\beta_{i}}}\right)+\gamma\left(\frac{2\left(P_{i}-\alpha_{i}\left(S_{p}{ }^{i}\right)^{-\beta_{i}-\beta}\right)^{3} \mu_{i}{ }^{4}}{\alpha_{i}\left(S_{p}{ }^{i}\right)^{-3 \beta_{i}}}\right. \\
& +\mu_{i}{ }^{4}-\frac{3\left(P_{i}-\alpha_{i}\left(S_{p}{ }^{i}\right)^{-\beta_{i}}\right) \mu_{i}{ }^{4}}{\alpha_{i}\left(S_{p}{ }^{i}\right)^{-\beta_{i}}}+\frac{3\left(P_{i}-\alpha_{i}\left(S_{p}{ }^{i}\right)^{-\beta_{i}}\right)^{5} \theta_{i} \mu_{i}^{5}}{\alpha_{i}\left(S_{p}{ }^{i}\right)^{-5 \beta_{i}}}+\frac{\theta_{i} \mu_{i}^{5}}{15}-\frac{20\left(P_{i}-\alpha_{i}\left(S_{p}{ }^{i}\right)^{-\beta_{i}}\right)^{3} \theta_{i} \mu_{i}^{5}}{\alpha_{i}\left(S_{p}{ }^{i}\right)^{-3 \beta_{i}}} \\
& \left.\left.+15\left(P_{i}-\alpha_{i}\left(S_{p}{ }^{i}\right)^{-\beta_{i}}\right) \theta_{i} \mu_{i}^{5}\right)\right\}-\frac{W_{i}}{\mu_{i}}\left(\frac{P_{i}}{\alpha_{i}\left(S_{p}{ }^{i}\right)^{-\beta_{i}}}-1\right)\left\{\mu_{i}{ }^{2}-30 \theta_{i} \mu_{i}^{3}-\left(P_{i}-\alpha_{i}\left(S_{p}{ }^{i}\right)^{-\beta_{i}}\right)\left(\frac{2 \mu_{i}^{3}}{m_{i} \alpha_{i}\left(S_{p}{ }^{i}\right)^{-\beta_{i}}}\right.\right. \\
& \left.\left.-\frac{4 \mu_{i}^{3}}{m_{i} \alpha_{i}\left(S_{p}^{i}\right)^{-\beta_{i}}}\right)\right\}-C_{S}^{i}\left\{\alpha_{i}\left(S_{p}^{i}\right)^{-\beta_{i}} m_{i}-\left(P_{i}-\alpha_{i}\left(S_{p}{ }^{i}\right)^{-\beta_{i}}\right) \mu_{i}+2\left(P_{i}-\alpha_{i}\left(S_{p}{ }^{i}\right)^{-\beta_{i}}\right) \theta_{i} \mu_{i}^{3}\right\} \\
& \left.-\frac{C_{L S}{ }^{i}(1-\delta) \alpha_{i}\left(S_{p}{ }^{i}\right)^{-\beta_{i}}}{\mu_{i}}\left\{\mu_{i}-\frac{\left(P_{i}-\alpha_{i}\left(S_{p}{ }^{i}\right)^{-\beta_{i}}\right)}{m_{i} \alpha_{i}\left(S_{p}{ }^{i}\right)^{-\beta_{i}}}\left(\mu_{i}{ }^{2}+\frac{\theta_{i} \mu_{i}{ }^{3}}{3}\right)\right\}-S_{U P}{ }^{i}\right]
\end{aligned}
$$

Consequently, one has to maximize the profit function:

$\operatorname{ETP}\left(P_{1}, P_{2} \ldots P_{n}\right)$

Subject to

$$
\begin{aligned}
& \sum_{i=1}^{n}\left(r_{i}+\frac{g_{i}}{P_{i}}+\alpha_{i} P_{i}\right) P_{i} \mu_{i} \leq C A P \\
& P_{1} \geq D_{1}, P_{2} \geq D_{2} \ldots . . P_{n} \geq D_{n}
\end{aligned}
$$

\subsection{Imprecise Model}

On applying the fuzzy parameters i.e. fuzzified holding cost, set up cost, shortages cost, lost sale cost. The expected total profit per breakdown is as follows,

$$
\begin{gathered}
\operatorname{ETP}\left(P_{1}, P_{2} \ldots P_{n}\right)=\sum_{i=1}^{n}\left[\left(S_{p}{ }^{i}-\left(r_{i}+\frac{g_{i}}{P_{i}}+H_{i} P_{i}\right)\right) P_{i} \mu_{i}-\frac{\left(P_{i}-\alpha_{i}\left(S_{p}{ }^{i}\right)^{-\beta_{i}}\right)}{\mu_{i}}\left\{\tilde{C}_{h}^{i}\left(\mu_{i}^{3}-2 \theta_{i} \mu_{i}{ }^{4}\right)+\gamma\left(2 \mu_{i}^{3}-8 \mu_{i}^{5}\right)\right\}\right. \\
-\alpha_{i}\left(S_{p}{ }^{i}\right)^{-\beta_{i}}\left\{\tilde { C } _ { h } ^ { i } \left(\frac{\left(P_{i}-\alpha_{i}\left(S_{p}{ }^{i}\right)^{-\beta_{i}}\right)^{2} \mu_{i}{ }^{3}}{\alpha_{i}\left(S_{p}{ }^{i}\right)^{-2 \beta_{i}}}-\frac{2\left(P_{i}-\alpha_{i}\left(S_{p}{ }^{i}\right)^{-\beta_{i}}\right) \mu_{i}{ }^{3}}{\alpha_{i}\left(S_{p}{ }^{i}\right)^{-\beta_{i}}}+\mu_{i}{ }^{3}-\frac{4\left(P_{i}-\alpha_{i}\left(S_{p}{ }^{i}\right)^{-\beta_{i}}\right)^{3} \theta_{i} \mu_{i}{ }^{4}}{\alpha_{i}\left(S_{p}{ }^{i}\right)^{-3 \beta_{i}}}\right.\right.
\end{gathered}
$$




$$
\begin{aligned}
& \left.-\frac{2\left(P_{i}-\alpha_{i}\left(S_{p}{ }^{i}\right)^{-\beta_{i}}\right)^{4} \theta_{i} \mu_{i}{ }^{4}}{\alpha_{i}\left(S_{p}{ }^{i}\right)^{-4 \beta_{i}}}+2 \theta_{i} \mu_{i}{ }^{4}-\frac{4\left(P_{i}-\alpha_{i}\left(S_{p}{ }^{i}\right)^{-\beta_{i}}\right) \theta_{i} \mu_{i}{ }^{4}}{\alpha_{i}\left(S_{p}{ }^{i}\right)^{-\beta_{i}}}\right)+\gamma\left(\frac{2\left(P_{i}-\alpha_{i}\left(S_{p}{ }^{i}\right)^{-\beta_{i}}\right)^{3} \mu_{i}{ }^{4}}{\alpha_{i}\left(S_{p}{ }^{i}\right)^{-3 \beta_{i}}}+\mu_{i}{ }^{4}\right. \\
& -\frac{3\left(P_{i}-\alpha_{i}\left(S_{p}{ }^{i}\right)^{-\beta_{i}}\right) \mu_{i}{ }^{4}}{\alpha_{i}\left(S_{p}{ }^{i}\right)^{-\beta_{i}}}+\frac{3\left(P_{i}-\alpha_{i}\left(S_{p}{ }^{i}\right)^{-\beta_{i}}\right)^{5} \theta_{i} \mu_{i}{ }^{5}}{\alpha_{i}\left(S_{p}{ }^{i}\right)^{-5 \beta_{i}}}+\frac{\theta_{i} \mu_{i}^{5}}{15}-\frac{20\left(P_{i}-\alpha_{i}\left(S_{p}{ }^{i}\right)^{-\beta_{i}}\right)^{3} \theta_{i} \mu_{i}{ }^{5}}{\alpha_{i}\left(S_{p}{ }^{i}\right)^{-3 \beta_{i}}} \\
& \left.\left.+15\left(P_{i}-\alpha_{i}\left(S_{p}{ }^{i}\right)^{-\beta_{i}}\right) \theta_{i} \mu_{i}{ }^{5}\right)\right\}-\frac{W_{i}}{\mu_{i}}\left(\frac{P_{i}}{\alpha_{i}\left(S_{p}{ }^{i}\right)^{-\beta_{i}}}-1\right)\left\{\mu_{i}{ }^{2}-30 \theta_{i} \mu_{i}{ }^{3}-\left(P_{i}-\alpha_{i}\left(S_{p}{ }^{i}\right)^{-\beta_{i}}\right)\right. \\
& \left.\left(\frac{2 \mu_{i}^{3}}{m_{i} \alpha_{i}\left(S_{p}{ }^{i}\right)^{-\beta_{i}}}-\frac{4 \mu_{i}^{3}}{m_{i} \alpha_{i}\left(S_{p}{ }^{i}\right)^{-\beta_{i}}}\right)\right\}-\tilde{C}_{s}^{i}\left\{\alpha_{i}\left(S_{p}{ }^{i}\right)^{-\beta_{i}} m_{i}-\left(P_{i}-\alpha_{i}\left(S_{p}{ }^{i}\right)^{-\beta_{i}}\right) \mu_{i}+2\left(P_{i}-\alpha_{i}\left(S_{p}{ }^{i}\right)^{-\beta_{i}}\right) \theta_{i} \mu_{i}{ }^{3}\right\} \\
& \left.-\frac{\tilde{C}_{L S}^{i}(1-\delta) \alpha_{i}\left(S_{p}{ }^{i}\right)^{-\beta_{i}}}{\mu_{i}}\left\{\mu_{i}-\frac{\left(P_{i}-\alpha_{i}\left(S_{p}{ }^{i}\right)^{-\beta_{i}}\right)}{m_{i} \alpha_{i}\left(S_{p}{ }^{i}\right)^{-\beta_{i}}}\left(\mu_{i}{ }^{2}+\frac{\theta_{i} \mu_{i}^{3}}{3}\right)\right\}-\tilde{S}_{U P}^{i}\right]
\end{aligned}
$$

where $(\sim)$ denotes the fuzzification of the parameters. In practical it is not easy to decide the holding cost for long period. Therefore, it becomes reasonable to locate holding cost in an interval $\left[C_{h}{ }^{i}-\Delta_{1}, C_{h}{ }^{i}+\Delta_{2}\right]$, where $0<\Delta_{1}<C_{h}{ }^{i}$ and $\Delta_{1}, \Delta_{2}>0$. The error in the value of holding cost will be larger if the deviation of it is farthest from $C_{h}{ }^{i}$ obviously error will attain its maximum at $C_{h}{ }^{i}-\Delta_{1}$ and $C_{h}{ }^{i}+\Delta_{2}$. In fuzzy system error is changed to the confidence level such as: if error is zero then the confidence level to be 1 . Maximum error is attained at $C_{h}{ }^{i}-\Delta_{1}$ and $C_{h}{ }^{i}+\Delta_{2}$ and then the confidence level is zero. If the value lies in the interval then the confidence level is any real value between zero and one. The above conditions are represented by the fuzzy triangular numbers.

$\widetilde{C_{h}^{l}}=\left[C_{h}^{i}-\Delta_{1}, C_{h}^{i}, C_{h}^{i}+\Delta_{2}\right]$,

where, $0<\Delta_{1}<C_{h}{ }^{i}, \Delta_{1}, \Delta_{2}>0$ and the signed distance of $C_{h}{ }^{i}$ is given by the relation

$d\left(\widetilde{C_{h}^{l}}, \tilde{0}\right)=C_{h}{ }^{i}+\frac{1}{4}\left(\Delta_{2}-\Delta_{1}\right)$

$d\left(\widetilde{C_{h}^{l}}, \widetilde{0}\right)>0$ and

$d\left(\widetilde{C_{h}^{l}}, \tilde{0}\right) \in\left[C_{h}{ }^{i}-\Delta_{1}, C_{h}{ }^{i}, C_{h}{ }^{i}+\Delta_{2}\right]$

$d\left(\widetilde{C_{h}^{l}}, \tilde{0}\right)$ is the estimated total holding cost during the planning period $[0, \mathrm{x}]$ based on the signed distance. In a perfect competitive situation, the shortages cost, lost sale cost and set up cost of a unit per unit time in a planning period $\mathrm{x}$ may also fluctuate from its actual value. Let us suppose that it lies in the interval $\left[C_{S}{ }^{i}-\Delta_{3}, C_{S}{ }^{i}+\Delta_{4}\right],\left[C_{L S}{ }^{i}-\Delta_{5}, C_{L S}{ }^{i}+\Delta_{6}\right]\left[S_{U P}{ }^{i}-\Delta_{7}, S_{U P}{ }^{i}+\Delta_{8}\right]$ as discussed above, we considered a fuzzy triangular number to represent the shortages cost, lost sale cost and set up cost as below:

$\widetilde{C_{S}^{l}}=\left[C_{S}^{i}-\Delta_{3}, C_{S}^{i}, C_{S}^{i}+\Delta_{4}\right]$,

$\widetilde{C_{L S}^{l}}=\left[C_{L S}^{i}-\Delta_{5}, C_{L S}^{i}, C_{L S}^{i}+\Delta_{6}\right]$,

$\widetilde{S_{U P}^{\tau}}=\left[S_{U P}^{i}-\Delta_{7}, S_{U P}^{i}, S_{U P}^{i}+\Delta_{8}\right]$,

where, $0<\Delta_{3}<C_{S}^{i}, 0<\Delta_{5}<C_{L S}^{i}, 0<\Delta_{7}<S_{U P}^{i}$ and $\Delta_{3}, \Delta_{4}>0, \Delta_{5}, \Delta_{6}>0$ and $\Delta_{7}, \Delta_{8}>0$

and the signed distance of $C_{S}^{i}, C_{L S}^{i}, C_{U P}^{i}$ is given by the formula

$d\left(\widetilde{C_{S}^{l}}, \tilde{0}\right)=C_{S}{ }^{i}+\frac{1}{4}\left(\Delta_{4}-\Delta_{3}\right)$,

$d\left(C_{S}{ }^{i}, \tilde{0}\right)>0$ and $d\left(C_{S}{ }^{i}, \tilde{0}\right) \in\left[C_{S}{ }^{i}-\Delta_{3}, C_{S}{ }^{i}, C_{S}{ }^{i}+\Delta_{4}\right]$, 
and $d\left(\widetilde{C_{L S}}, \tilde{0}\right)=C_{L S}{ }^{i}+\frac{1}{4}\left(\Delta_{6}-\Delta_{5}\right)$,

$d\left(\widetilde{C_{L S}^{\tau}}, \tilde{0}\right)>0$ and $d\left(\tilde{C}_{L S}{ }^{i}, \tilde{0}\right) \in\left[C_{L S}{ }^{i}-\Delta_{5}, C_{L S}{ }^{i}, C_{L S}{ }^{i}+\Delta_{6}\right]$,

and $d\left(\widetilde{S_{U P}^{\tau}}, \tilde{0}\right)=S_{U P}^{i}+\frac{1}{4}\left(\Delta_{8}-\Delta_{7}\right)$,

$d\left(\widetilde{S_{U P}^{\tau}}, \tilde{0}\right)>0$ and $d\left(\widetilde{S_{U P}}, \tilde{0}\right) \in\left[S_{U P}{ }^{i}-\Delta_{7}, S_{U P}{ }^{i}, S_{U P}{ }^{i}+\Delta_{8}\right]$

From Eq. (13), we have

$F\left(\tilde{C}_{h}^{i}, \tilde{C}_{S}^{i}, \tilde{C}_{L S}^{i}, \tilde{S}_{U P}^{i}\right)=\operatorname{ETP}\left(P_{1}, P_{2} \ldots P_{n}\right)$

Now by the fuzzy triangular rule, we have

$F\left(\tilde{C}_{h}^{i}, \tilde{C}_{S}^{i}, \tilde{C}_{L S}^{i}, \tilde{S}_{U P}^{i}\right)=\left(F_{1}, F_{2} \ldots F_{n}\right)$

$F\left(\tilde{C}_{h}^{i}, \tilde{C}_{S}^{i}, \tilde{C}_{L S}^{i}, \tilde{S}_{U P}^{i}\right)$ is the estimated fuzzified total cost and $\mathrm{F}_{1}, \mathrm{~F}_{2}, \mathrm{~F}_{3}$ are obtained as:

$$
\begin{aligned}
& F_{1}=\sum_{i=1}^{n}\left[\left(S_{p}^{i}-\left(r_{i}+\frac{g_{i}}{P_{i}}+H_{i} P_{i}\right)\right) P_{i} \mu_{i}-\frac{\left(P_{i}-\alpha_{i}\left(S_{p}{ }^{i}\right)^{-\beta_{i}}\right)}{\mu_{i}}\left\{\left(C_{h}{ }^{i}-\Delta_{1}\right)\left(\mu_{i}^{3}-2 \theta_{i} \mu_{i}^{4}\right)+\gamma\left(2 \mu_{i}^{3}-8 \mu_{i}{ }^{5}\right)\right\}\right. \\
& -\alpha_{i}\left(S_{p}{ }^{i}\right)^{-\beta_{i}}\left\{( C _ { h } { } ^ { i } - \Delta _ { 1 } ) \left(\frac{\left(P_{i}-\alpha_{i}\left(S_{p}{ }^{i}\right)^{-\beta_{i}}\right)^{2} \mu_{i}{ }^{3}}{\alpha_{i}\left(S_{p}{ }^{i}\right)^{-2 \beta_{i}}}-\frac{2\left(P_{i}-\alpha_{i}\left(S_{p}{ }^{i}\right)^{-\beta_{i}}\right) \mu_{i}{ }^{3}}{\alpha_{i}\left(S_{p}{ }^{i}\right)^{-\beta_{i}}}+\mu_{i}{ }^{3}-\frac{4\left(P_{i}-\alpha_{i}\left(S_{p}{ }^{i}\right)^{-\beta_{i}}\right)^{3} \theta_{i} \mu_{i}{ }^{4}}{\alpha_{i}\left(S_{p}{ }^{i}\right)^{-3 \beta_{i}}}\right.\right. \\
& \left.-\frac{2\left(P_{i}-\alpha_{i}\left(S_{p}^{i}\right)^{-\beta_{i}}\right)^{4} \theta_{i} \mu_{i}{ }^{4}}{\alpha_{i}\left(S_{p}^{i}\right)^{-4 \beta_{i}}}+2 \theta_{i} \mu_{i}^{4}-\frac{4\left(P_{i}-\alpha_{i}\left(S_{p}^{i}\right)^{-\beta_{i}}\right) \theta_{i} \mu_{i}{ }^{4}}{\alpha_{i}\left(S_{p}^{i}\right)^{-\beta_{i}}}\right)+\gamma\left(\frac{2\left(P_{i}-\alpha_{i}\left(S_{p}^{i}\right)^{-\beta_{i}}\right)^{3} \mu_{i}{ }^{4}}{\alpha_{i}\left(S_{p}^{i}\right)^{-3 \beta_{i}}}+\mu_{i}^{4}\right. \\
& -\frac{3\left(P_{i}-\alpha_{i}\left(S_{p}^{i}\right)^{-\beta_{i}}\right) \mu_{i}^{4}}{\alpha_{i}\left(S_{p}^{i}\right)^{-\beta_{i}}}+\frac{3\left(P_{i}-\alpha_{i}\left(S_{p}^{i}\right)^{-\beta_{i}}\right)^{5} \theta_{i} \mu_{i}^{5}}{\alpha_{i}\left(S_{p}^{i}\right)^{-\beta_{i}}}+\frac{\theta_{i} \mu_{i}^{5}}{15}-\frac{20\left(P_{i}-\alpha_{i}\left(S_{p}^{i}\right)^{-\beta_{i}}\right)^{3} \theta_{i} \mu_{i}^{5}}{\alpha_{i}\left(S_{p}^{i}\right)^{-3 \beta_{i}}} \\
& \left.\left.+15\left(P_{i}-\alpha_{i}\left(S_{p}{ }^{i}\right)^{-\beta_{i}}\right) \theta_{i} \mu_{i}^{5}\right)\right\}-\frac{W_{i}}{\mu_{i}}\left(\frac{P_{i}}{\alpha_{i}\left(S_{p}{ }^{i}\right)^{-\beta_{i}}}-1\right)\left\{\mu_{i}{ }^{2}-30 \theta_{i} \mu_{i}^{3}-\left(P_{i}-\alpha_{i}\left(S_{p}{ }^{i}\right)^{-\beta_{i}}\right)\right. \\
& \left.\left(\frac{2 \mu_{i}^{3}}{m_{i} \alpha_{i}\left(S_{p}{ }^{i}\right)^{-\beta_{i}}}-\frac{4 \mu_{i}{ }^{3}}{m_{i} \alpha_{i}\left(S_{p}{ }^{i}\right)^{-\beta_{i}}}\right)\right\}-\left(C_{S}{ }^{i}-\Delta_{3}\right)\left\{\alpha_{i}\left(S_{p}{ }^{i}\right)^{-\beta_{i}} m_{i}-\left(P_{i}-\alpha_{i}\left(S_{p}{ }^{i}\right)^{-\beta_{i}}\right) \mu_{i}\right. \\
& \left.+2\left(P_{i}-\alpha_{i}\left(S_{p}{ }^{i}\right)^{-\beta_{i}}\right) \theta_{i} \mu_{i}{ }^{3}\right\}-\frac{\left(C_{L S}{ }^{i}-\Delta_{5}\right)(1-\delta) \alpha_{i}\left(S_{p}{ }^{i}\right)^{-\beta_{i}}}{\mu_{i}}\left\{\mu_{i}-\frac{\left(P_{i}-\alpha_{i}\left(S_{p}{ }^{i}\right)^{-\beta_{i}}\right)}{m_{i} \alpha_{i}\left(S_{p}{ }^{i}\right)^{-\beta_{i}}}\right. \\
& \left.\left.\left(\mu_{i}^{2}+\frac{\theta_{i} \mu_{i}^{3}}{3}\right)\right\}-\left(S_{U P}^{i}-\Delta_{7}\right)\right] \\
& F_{2}=F_{\left(P_{1}, P_{2} \ldots P_{n}\right)}\left(C_{h}{ }^{i}, C_{S}{ }^{i}, C_{L S}{ }^{i}, S_{U P}{ }^{i}\right) \\
& F_{3}=\sum_{i=1}^{n}\left[\left(S_{p}^{i}-\left(r_{i}+\frac{g_{i}}{P_{i}}+H_{i} P_{i}\right)\right) P_{i} \mu_{i}-\frac{\left(P_{i}-\alpha_{i}\left(S_{p}^{i}\right)^{-\beta_{i}}\right)}{\mu_{i}}\left\{\left(C_{h}^{i}+\Delta_{2}\right)\left(\mu_{i}^{3}-2 \theta_{i} \mu_{i}^{4}\right)+\gamma\left(2 \mu_{i}^{3}-8 \mu_{i}^{5}\right)\right\}\right. \\
& -\alpha_{i}\left(S_{p}{ }^{i}\right)^{-\beta_{i}}\left\{( C _ { h } ^ { i } + \Delta _ { 2 } ) \left(\frac{\left(P_{i}-\alpha_{i}\left(S_{p}{ }^{i}\right)^{-\beta_{i}}\right)^{2} \mu_{i}^{3}}{\alpha_{i}\left(S_{p}{ }^{i}\right)^{-2 \beta_{i}}}-\frac{2\left(P_{i}-\alpha_{i}\left(S_{p}{ }^{i}\right)^{-\beta_{i}}\right) \mu_{i}^{3}}{\alpha_{i}\left(S_{p}{ }^{i}\right)^{-\beta_{i}}}+\mu_{i}^{3}-\frac{4\left(P_{i}-\alpha_{i}\left(S_{p}{ }^{i}\right)^{-\beta_{i}}\right)^{3} \theta_{i} \mu_{i}^{4}}{\alpha_{i}\left(S_{p}{ }^{i}\right)^{-3 \beta_{i}}}\right.\right. \\
& \left.-\frac{2\left(P_{i}-\alpha_{i}\left(S_{p}^{i}\right)^{-\beta_{i}}\right)^{4} \theta_{i} \mu_{i}{ }^{4}}{\alpha_{i}\left(S_{p}{ }^{i}\right)^{-4 \beta_{i}}}+2 \theta_{i} \mu_{i}{ }^{4}-\frac{4\left(P_{i}-\alpha_{i}\left(S_{p}{ }^{i}\right)^{-\beta_{i}}\right) \theta_{i} \mu_{i}{ }^{4}}{\alpha_{i}\left(S_{p}{ }^{i}\right)^{-\beta_{i}}}\right)+\gamma\left(\frac{2\left(P_{i}-\alpha_{i}\left(S_{p}{ }^{i}\right)^{-\beta_{i}}\right)^{3} \mu_{i}^{4}}{\alpha_{i}\left(S_{p}{ }^{i}\right)^{-3 \beta_{i}}}+\mu_{i}{ }^{4}\right.
\end{aligned}
$$




$$
\begin{aligned}
& -\frac{3\left(P_{i}-\alpha_{i}\left(S_{p}{ }^{i}\right)^{-\beta_{i}}\right) \mu_{i}^{4}}{\alpha_{i}\left(S_{p}{ }^{i}\right)^{-\beta_{i}}}+\frac{3\left(P_{i}-\alpha_{i}\left(S_{p}{ }^{i}\right)^{-\beta_{i}}\right)^{5} \theta_{i} \mu_{i}^{5}}{\alpha_{i}\left(S_{p}{ }^{i}\right)^{-\beta_{i}}}+\frac{\theta_{i} \mu_{i}^{5}}{15}-\frac{20\left(P_{i}-\alpha_{i}\left(S_{p}{ }^{i}\right)^{-\beta_{i}}\right)^{3} \theta_{i} \mu_{i}{ }^{5}}{\alpha_{i}\left(S_{p}{ }^{i}\right)^{-3 \beta_{i}}} \\
& \left.\left.+15\left(P_{i}-\alpha_{i}\left(S_{p}{ }^{i}\right)^{-\beta_{i}}\right) \theta_{i} \mu_{i}{ }^{5}\right)\right\}-\frac{W_{i}}{\mu_{i}}\left(\frac{P_{i}}{\alpha_{i}\left(S_{p}{ }^{i}\right)^{-\beta_{i}}}-1\right)\left\{\mu_{i}{ }^{2}-30 \theta_{i} \mu_{i}^{3}-\left(P_{i}-\alpha_{i}\left(S_{p}{ }^{i}\right)^{-\beta_{i}}\right)\right. \\
& \left.\quad\left(\frac{2 \mu_{i}^{3}}{m_{i} \alpha_{i}\left(S_{p}{ }^{i}\right)^{-\beta_{i}}}-\frac{4 \mu_{i}^{3}}{m_{i} \alpha_{i}\left(S_{p}{ }^{i}\right)^{-\beta_{i}}}\right)\right\}-\left(C_{S}{ }^{i}+\Delta_{4}\right)\left\{\alpha_{i}\left(S_{p}{ }^{i}\right)^{-\beta_{i}} m_{i}-\left(P_{i}-\alpha_{i}\left(S_{p}{ }^{i}\right)^{-\beta_{i}}\right) \mu_{i}\right. \\
& \left.+2\left(P_{i}-\alpha_{i}\left(S_{p}{ }^{i}\right)^{-\beta_{i}}\right) \theta_{i} \mu_{i}{ }^{3}\right\}-\frac{\left(C_{L S}{ }^{i}+\Delta_{6}\right)(1-\delta) \alpha_{i}\left(S_{p}{ }^{i}\right)^{-\beta_{i}}}{\mu_{i}}\left\{\mu_{i}-\frac{\left(P_{i}-\alpha_{i}\left(S_{p}{ }^{i}\right)^{-\beta_{i}}\right)}{m_{i} \alpha_{i}\left(S_{p}{ }^{i}\right)^{-\beta_{i}}}\right. \\
& \left.\left.\left(\mu_{i}{ }^{2}+\frac{\theta_{i} \mu_{i}{ }^{3}}{3}\right)\right\}-\left(S_{U P}{ }^{i}+\Delta_{8}\right)\right]
\end{aligned}
$$

Defuzzification of fuzzy number $F_{\left(P_{1}, P_{2} \ldots P_{n}\right)}\left(\tilde{C}_{h}^{i}, \tilde{C}_{S}^{i}, \tilde{C}_{L S}^{i}, \tilde{S}_{U P}^{i}\right)$ using signed distance method is given by:

$$
\begin{aligned}
& F_{\left(P_{1}, P_{2} \ldots P_{n}\right)}\left(\tilde{C}_{h}^{i}, \tilde{C}_{S}^{i}, \tilde{C}_{L S}^{i}, \tilde{S}_{U P}^{i}, \tilde{0}\right)=F_{\left(P_{1}, P_{2} \ldots P_{n}\right)}\left(C_{h}^{i}, C_{S}{ }^{i}, C_{L S}{ }^{i}, S_{U P}{ }^{i}\right)+\frac{1}{4} \sum_{i=1}^{n}\left[-\frac{\left(P_{i}-\alpha_{i}\left(S_{p}{ }^{i}\right)^{-\beta_{i}}\right)}{\mu_{i}}\right. \\
& \left(\Delta_{2}+\Delta_{1}\right)\left(\mu_{i}^{3}-2 \theta_{i} \mu_{i}^{4}\right)-\alpha_{i}\left(S_{p}^{i}\right)^{-\beta_{i}}\left(\Delta_{2}+\Delta_{1}\right)\left(\frac{\left(P_{i}-\alpha_{i}\left(S_{p}{ }^{i}\right)^{-\beta_{i}}\right)^{2} \mu_{i}^{3}}{\alpha_{i}\left(S_{p}{ }^{i}\right)^{-2 \beta_{i}}}\right. \\
& -\frac{2\left(P_{i}-\alpha_{i}\left(S_{p}{ }^{i}\right)^{-\beta_{i}}\right) \mu_{i}{ }^{3}}{\alpha_{i}\left(S_{p}{ }^{i}\right)^{-\beta_{i}}}+\mu_{i}{ }^{3}-\frac{4\left(P_{i}-\alpha_{i}\left(S_{p}{ }^{i}\right)^{-\beta_{i}}\right)^{3} \theta_{i} \mu_{i}{ }^{4}}{\alpha_{i}\left(S_{p}{ }^{i}\right)^{-3 \beta_{i}}}-\frac{2\left(P_{i}-\alpha_{i}\left(S_{p}{ }^{i}\right)^{-\beta_{i}}\right)^{4} \theta_{i} \mu_{i}{ }^{4}}{\alpha_{i}\left(S_{p}{ }^{i}\right)^{-4 \beta_{i}}} \\
& \left.+2 \theta_{i} \mu_{i}^{4}-\frac{4\left(P_{i}-\alpha_{i}\left(S_{p}^{i}\right)^{-\beta_{i}}\right) \theta_{i} \mu_{i}^{4}}{\alpha_{i}\left(S_{p}{ }^{i}\right)^{-\beta_{i}}}\right)-\left(\Delta_{4}+\Delta_{3}\right)\left\{\alpha_{i}\left(S_{p}^{i}\right)^{-\beta_{i}} m_{i}-\left(P_{i}-\alpha_{i}\left(S_{p}^{i}\right)^{-\beta_{i}}\right) \mu_{i}\right. \\
& \left.+2\left(P_{i}-\alpha_{i}\left(S_{p}^{i}\right)^{-\beta_{i}}\right) \theta_{i} \mu_{i}^{3}\right\}-\left(\Delta_{6}+\Delta_{5}\right) \frac{(1-\delta) \alpha_{i}\left(S_{p}{ }^{i}\right)^{-\beta_{i}}}{\mu_{i}}\left\{\mu_{i}-\frac{\left(P_{i}-\alpha_{i}\left(S_{p}{ }^{i}\right)^{-\beta_{i}}\right)}{m_{i} \alpha_{i}\left(S_{p}{ }^{i}\right)^{-\beta_{i}}}\right. \\
& \left.\left.\left(\mu_{i}^{2}+\frac{\theta_{i} \mu_{i}^{3}}{3}\right)\right\}-\left(\Delta_{8}+\Delta_{7}\right)\right]
\end{aligned}
$$

\begin{tabular}{|c|c|c|c|c|c|c|c|}
\hline Item ' $\mathrm{i}$ ' & $\mu_{\mathrm{i}}$ & $r_{i}$ & $g_{i}$ & $\mathrm{H}_{\mathrm{i}}$ & $\alpha_{i}$ & $\beta_{\mathrm{i}}$ & $C_{L S}{ }^{i}$ \\
\hline 1 & 5 & 250 & 2500 & 0.02 & 8500 & 2.0 & 26 \\
\hline 2 & 6 & 200 & 2400 & 0.03 & 8000 & 2.2 & 28 \\
\hline Item ' $i$ ' & $\theta_{\mathrm{i}}$ & $\mathrm{W}_{\mathrm{i}}$ & $\mathrm{m}_{\mathrm{i}}$ & $S_{P}^{i}$ & $C_{S}^{i}$ & $C_{h}^{i}$ & \\
\hline 1 & 0.07 & 40 & 0.65 & 60 & 14 & 15 & \\
\hline 2 & 0.06 & 45 & 0.75 & 50 & 17 & 15 & \\
\hline$\Delta_{1}$ & $\Delta_{2}$ & $\Delta_{3}$ & $\Delta_{4}$ & $\Delta_{5}$ & $\Delta_{6}$ & $\Delta_{7}$ & $\Delta_{8}$ \\
\hline 2 & 3 & 4 & 3.5 & 2.5 & 3 & 4.5 & 5 \\
\hline
\end{tabular}

Eq. (31) gives an estimate of profit function. For maximization of profit function, differentiate the Eq. (31) with respect to $\mathrm{P}_{1}, \mathrm{P}_{2} \ldots \mathrm{P}_{\mathrm{n}}$. The results are illustrated numerically with the help of the software Mathematica5.2.

\section{Numerical Illustrations}

The model has been explored numerically as well. There are following data which can be used to explain the model, based on the studies in proper unit. We have studied for two items. The following numerical study has been used to find the optimal solution of the multi items production model.

The common input parameters are: $\gamma=0.6, \delta=0.4$, 
The optimal values of $\mathrm{P}_{1}$ and $\mathrm{P}_{2}$ are 7.51556 and 5.91907 respectively. The optimal net profit is 136989.

\section{Sensitivity Analysis}

The sensitivity analysis is presented due to the changes in the fuzzy parameters for the production and profit.

Table 1

Sensitivity Analysis of Optimal Solution w.r.t. System Parameters

\begin{tabular}{cllccccc}
\hline Parameter & \% Change & $-30 \%$ & $-20 \%$ & $-10 \%$ & $10 \%$ & $20 \%$ & $30 \%$ \\
\hline \multirow{3}{*}{$\Delta_{1}$} & $\mathrm{P}_{1}$ & +1.1861 & +0.7929 & +0.3976 & -0.3998 & -0.8022 & -1.2071 \\
& $\mathrm{P}_{2}$ & +0.4651 & +0.3100 & +0.1551 & -0.1551 & -0.3102 & -0.4653 \\
& Profit & +4.6055 & +3.055 & +1.5198 & -1.5052 & -2.9951 & -4.4697 \\
\hline \multirow{3}{*}{$\Delta_{2}$} & $\mathrm{P}_{1}$ & -1.8192 & -1.2071 & -0.6008 & +0.5956 & +1.1861 & +1.7703 \\
& $\mathrm{P}_{2}$ & -0.6981 & -0.4653 & -0.2326 & +0.2325 & +0.4651 & +0.6974 \\
& Profit & -6.6538 & -4.4697 & -2.2520 & +2.2856 & +4.6055 & +6.9604 \\
\hline \multirow{3}{*}{$\Delta_{3}$} & $\mathrm{P}_{1}$ & -0.0049 & -0.0033 & -0.0016 & +0.0017 & +0.0033 & +0.0051 \\
& $\mathrm{P}_{2}$ & -0.0007 & -0.0005 & -0.0003 & +0.0002 & +0.0005 & +0.0007 \\
& Profit & -0.0343 & -0.0234 & -0.0117 & +0.0109 & +0.0226 & +0.0336 \\
\hline \multirow{4}{*}{$\Delta_{4}$} & $\mathrm{P}_{1}$ & +0.0044 & +0.0029 & +0.0015 & -0.0015 & -0.0028 & -0.0043 \\
& $\mathrm{P}_{2}$ & +0.0007 & +0.0003 & +0.0002 & -0.0002 & -0.0005 & -0.0007 \\
& Profit & +0.0292 & +0.0197 & +0.0095 & -0.0102 & -0.0204 & -0.0299 \\
\hline \multirow{4}{*}{$\Delta_{5}$} & $\mathrm{P}_{1}$ & +0.0013 & +0.0009 & +0.0005 & -0.0004 & -0.0008 & -0.0012 \\
& $\mathrm{P}_{2}$ & +0.0002 & 0.0 & 0.0 & 0.0 & -0.0002 & -0.0002 \\
& Profit & +0.0066 & +0.0044 & +0.0022 & -0.0022 & -0.0044 & -0.0066 \\
\hline \multirow{3}{*}{$\Delta_{6}$} & $\mathrm{P}_{1}$ & -0.0015 & -0.0009 & -0.0005 & +0.0005 & +0.0011 & +0.0016 \\
& $\mathrm{P}_{2}$ & -0.0002 & -0.0002 & 0.0 & 0.0 & 0.0 & +0.0002 \\
& Profit & -0.0080 & -0.0058 & -0.0029 & +0.0022 & +0.0051 & +0.0073 \\
\hline
\end{tabular}

\section{Observations}

From the sensitivity analysis, it has been observed that

1. $\Delta_{1}$ as increases, $P_{1}, P_{2}$ and the profit are decreases and $\Delta_{1}$ as decreases, $P_{1}, P_{2}$ and the profit are increases. The graphical representation of $\mathrm{P}_{1}, \mathrm{P}_{2}$ and the profit w.r.t. $\Delta_{1}$ is illustrated by figure 1 .

2. As $\Delta_{2}$ increases, $\mathrm{P}_{1}, \mathrm{P}_{2}$ and the profit are increases and as $\Delta_{2}$ decreases, $\mathrm{P}_{1}, \mathrm{P}_{2}$ and the profit are decreases. The variation of $\mathrm{P}_{1}, \mathrm{P}_{2}$ and the profit w.r.t. $\Delta_{2}$ is shown by figure 2 .

3. On increasing the $\Delta_{3}, \mathrm{P}_{1}, \mathrm{P}_{2}$ and the profit are increases and on decreasing the $\Delta_{3}, \mathrm{P}_{1}, \mathrm{P}_{2}$ and the profit are decreases. Graphically, it is shown by figure 3.

4. If $\Delta_{4}$ is increases then $\mathrm{P}_{1}, \mathrm{P}_{2}$ and the profit are decreases and if $\Delta_{4}$ is decreases then $\mathrm{P}_{1}, \mathrm{P}_{2}$ and the profit are increases. Figure 4 represented the variation of $\mathrm{P}_{1}, \mathrm{P}_{2}$ and the profit w.r.t. ' $\Delta_{4}$ '.

5. As $\Delta_{5}$ increases, $\mathrm{P}_{1}$ and the profit are decreases and as $\Delta_{5}$ decreases, $\mathrm{P}_{1}$ and the profit are increases and $\mathrm{P}_{2}$ is almost constant to change in $\Delta_{\mathbf{5}}$. It is shown by the figure 5 .

6. If $\Delta_{6}$ is increases then $P_{1}$ and the profit are increases and if $\Delta_{6}$ is decreases, $P_{1}$ and the profit are decreases and $\mathrm{P}_{2}$ is almost stable to change in $\Delta_{6}$. Figure 6 represented the variation of $\mathrm{P}_{1}, \mathrm{P}_{2}$ and the profit w.r.t. ' $\Delta_{\mathbf{6}}$ '. 


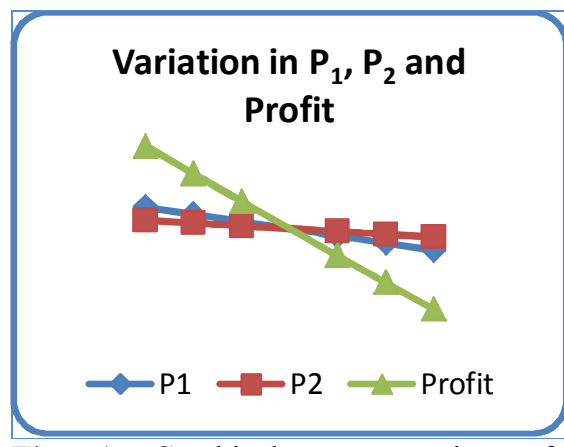

Fig. 1. Graphical representation of sensitivity of the $\mathrm{P}_{1}, \mathrm{P}_{2}$ and Profit w.r.t. $\Delta_{1}$

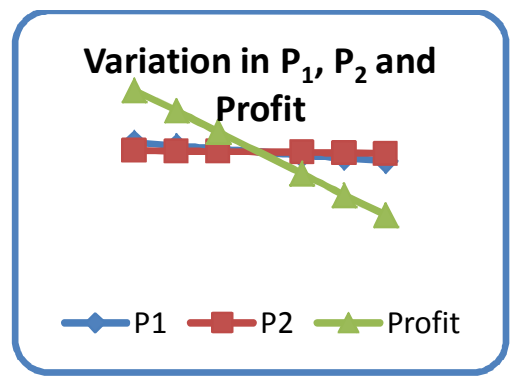

Fig. 4. Graphical representation of sensitivity of the $\mathrm{P}_{1}, \mathrm{P}_{2}$ and Profit w.r.t. $\Delta_{4}$

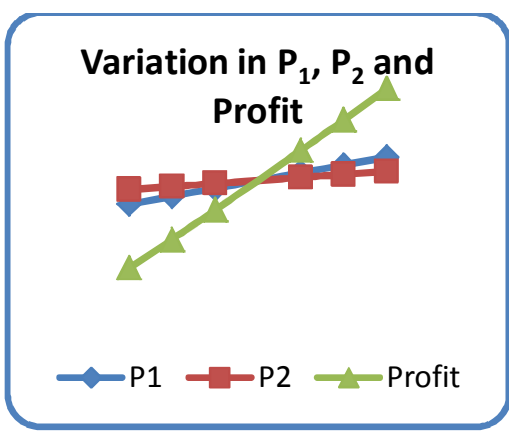

Fig. 2. Graphical representation of sensitivity of the $\mathrm{P}_{1}, \mathrm{P}_{2}$ and Profit w.r.t. $\Delta_{2}$

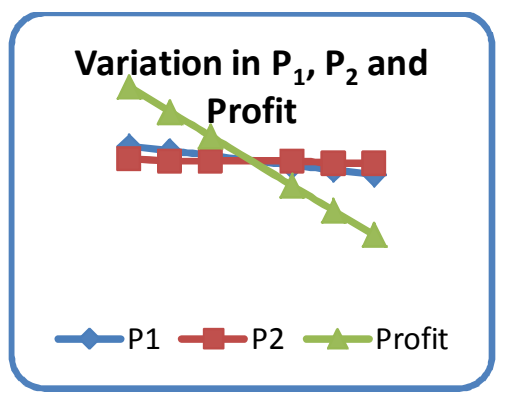

Fig. 5. Graphical representation of sensitivity of the $\mathrm{P}_{1}, \mathrm{P}_{2}$ and Profit w.r.t. $\Delta_{5}$

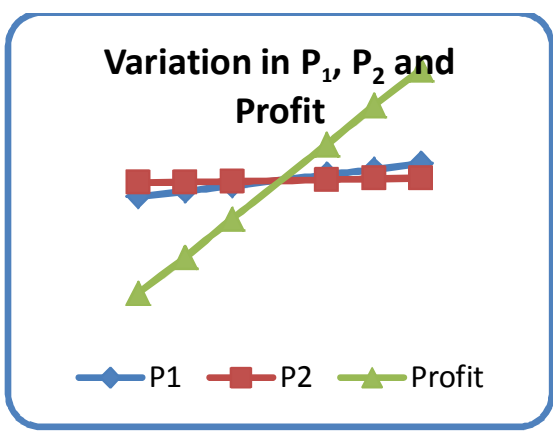

Fig. 3. Graphical representation of sensitivity of the $\mathrm{P}_{1}, \mathrm{P}_{2}$ and Profit w.r.t. $\Delta_{3}$

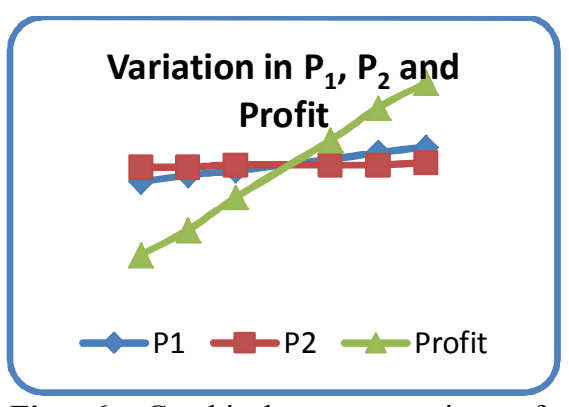

Fig. 6. Graphical representation of sensitivity of the $\mathrm{P}_{1}, \mathrm{P}_{2}$ and Profit w.r.t. $\Delta_{6}$

\section{Conclusions}

In this paper, an EMQ multi items model for unreliable production systems with the process of deterioration and machine breakdown has been discussed. In a competitive market, price of goods plays an important factor. Generally, a reduced price encourages a customer to buy more. For fitting in with realistic circumstances, the environment of the whole study has been taken as fuzzified. Model has been solved numerically with sensitivity analysis. All these facts have been shown graphically.

We have many real life situations in which multi items inventories are required. For instance: a pharmacist keeps a number of medicines of different brands, readymade clothes shop keeps dresses of different things in different colors and in different size, shoe store stocks shoes of various models and sizes. The presented model is much more realistic and practical.

\section{References}

Bellman, R.E., \& Zadeh, L.A. (1970). Decision making in a fuzzy environment. Management Science, 17, B141-B164.

Chakraborty, T., Giri, B.C., \& Chaudhuri, K.S. (2008). Production lot sizing with process deterioration and machine breakdown. European Journal of Operational Research, 185, 606-618.

Chakraborty, T., Giri, B.C., \& Chaudhuri, K.S. (2009). Production lot sizing with process deterioration and machine breakdown under inspection schedule. Omega, 37, 257- 271.

Dey, J.K., Kar, S., \& Maiti, M. (2005). An interactive method for inventory control with fuzzy leadtime and dynamic demand. European Journal of Operational Research, 167, 381-397.

Giri, B.C., \& Dohi, T. (2005). Exact formulation of stochastic EMQ model for an unreliable production systems. Journal of the Operational Research Society, 56 (5), 563-575.

Goyal, S.K. (1991). Economic production of a product and replenishment of its raw materials. Engineering Costs and Production Economics, 21, 111- 115. 
Goyal, S.K., \& Giri, B.C. (2003). The production-inventory problem of a product with time varying demand, production and deterioration rates. European Journal of Operational Research, 147, 549557.

Hsu, L.-F. (2012). A note on "an economic order quantity (EOQ) for items with imperfect quality and inspection errors". International Journal of Industrial Engineering Computations, 3(4), 695-702

Mahapatra, N.K., \& Maiti, M. (2005). Decision process for multi-objective, multi-item productioninventory system via interactive fuzzy satisficing technique. Computers and Mathematics with Applications, 49, 805-821.

Mahata, G. C., \& Mahata, P. (2011). Analysis of a fuzzy economic order quantity model for deteriorating items under retailer partial trade credit financing in a supply chain. Mathematical and Computer Modelling, 53(9-10), 1621-1636.

Maity, K., \& Maiti, M. (2008). A numerical approach to a multi-objective optimal inventory control problem for deteriorating multi-items under fuzzy inflation and discounting. Computers and Mathematics with Applications, 55, 1794-1807.

Padmanabhan, G., \& Vrat, P. (1990). Analysis of multi-systems under resource constraint, a nonlinear goal programming approach. Engineering Cost and Production Management, 13, 104-112.

Roy, T.K., \& Maiti, M. (1998). Multi-objective inventory models of deteriorating items with some constraints in a fuzzy environment. Computers and Operations Research, 25, 1085-1095.

Roy, T.K., \& Maiti, M. (2000). A multi-item fuzzy displayed inventory model under limited shelfspace. The International Journal of Fuzzy Mathematics, 8, 881-888.

Shukla, H., Shukla, V \& Yadava, S. (2013). EOQ model for deteriorating items with exponential demand rate and shortages. Uncertain Supply Chain Management, 1(2), 67-76.

Singh, S. R., Singh, A. P., \& Bhatia, D. (2010). A Supply Chain Model with Variable Holding Cost for Flexible Manufacturing System. International Journal of Operations Research and Optimization, 1, 107-120.

Singh, S.R., \& Singh, C. (2008). Fuzzy inventory model for finite rate of replenishment using signed distance method. International Transactions in Mathematical Sciences and Computer, 1, 1, 21-30.

Singh, S.R., Prasher, L., \& Saxena, N. (2013). A centralized reverse channel structure with flexible manufacturing under the stock out situation. International Journal of Industrial Engineering Computations, 4(4), 559-570.

Srinivasan, M.M., \& Lee, H.S. (1996). Production-inventory systems with preventive maintenance. IIE Transactions, 28, 879-890.

Tse, E., \& Makis, V. (1994). Optimization of the lot size and the time to replacement in a production system subject to random failure. In: Proceedings of the Third International Conference on Auto Tech, Taipei, Taiwan, 163-169.

Wang L., Fu, Q. L., \& Zeng, Y. R. (2012). Continuous review inventory models with a mixture of backorders and lost sales under fuzzy demand and different decision situations, Expert Systems with Applications, 39(4), 4181-4189.

Wee, H.M., \& Chung, S.L. (2005). An optimal production model for disposing excessive deteriorating stocks. Journal of the Chinese Institute of Industrial Engineers, 22(5), 369-378.

$\mathrm{Xu}, \mathrm{R} .$, \& Zhai, X. (2010). Manufacturer's coordination mechanism for single-period supply chain problems with fuzzy demand. Mathematical and Computer Modelling, 51(5-6), 693-699.

Yao, J.S., \& Wu, K. (1999). Consumer surplus and producer surplus for fuzzy demand and fuzzy supply. Fuzzy Sets and Systems, 103, 421-426.

Zadeh, L.A. (1965). Fuzzy sets. Information and Control, 8, 338-356.

Zimmermann, H.J. (1985). Description and optimization of fuzzy system. International Journal of General Systems, 2, 209-215. 